\title{
Reactive cutaneous capillary endothelial proliferation in advanced hepatocellular carcinoma patients treated with camrelizumab: data derived from a multicenter phase 2 trial
}

Feng Wang ${ }^{1,2+}$, Shukui Qin ${ }^{2 *}$, Xinchen Sun ${ }^{1 *}$, Zhenggang Ren ${ }^{3}$, Zhiqiang Meng ${ }^{4}$, Zhendong Chen ${ }^{5}$, Xiaoli Chai ${ }^{6}$, Jianping Xiong ${ }^{7}$, Yuxian Bai ${ }^{8}$, Lin Yang ${ }^{9}$, Hong Zhu ${ }^{10}$, Weijia Fang ${ }^{11}$, Xiaoyan Lin ${ }^{12}$, Xiaoming Chen ${ }^{13}$, Enxiao Li ${ }^{14}$, Linna Wang ${ }^{15}$, Ping Yan ${ }^{15}$ and Jianjun Zou ${ }^{15}$

\begin{abstract}
Background: Association of immune-related adverse events with tumor response has been reported. Reactive cutaneous capillary endothelial proliferation (RCCEP) is the most common adverse event related to camrelizumab, an immune checkpoint inhibitor, but lack of comprehensive analyses. In this study, we conducted comprehensive analyses on RCCEP in advanced hepatocellular carcinoma (HCC) patients treated with camrelizumab monotherapy.

Methods: Data were derived from a Chinese nationwide, multicenter phase 2 trial of camrelizumab in pre-treated advanced HCC. The occurrence, clinicopathological characteristics, and prognostic value of RCCEP were analyzed.

\footnotetext{
*Correspondence: qinsk@csco.org.cn; sunxinchen@csco.org.cn

${ }^{\dagger}$ Feng Wang and Shukui Qin contributed equally to this work.

${ }^{2}$ Department of Medical Oncology, Cancer Center of Jinling Hospital, No. 34, 34 Biao, Yanggongjing Street, Nanjing 210002, China

'Department of Radiotherapy, The First Affiliated Hospital of Nanjing Medical College, No. 300, Guangzhou Rode, Nanjing 210029, China

Full list of author information is available at the end of the article
}

(c) The Author(s). 2020 Open Access This article is licensed under a Creative Commons Attribution 4.0 International License, which permits use, sharing, adaptation, distribution and reproduction in any medium or format, as long as you give appropriate credit to the original author(s) and the source, provide a link to the Creative Commons licence, and indicate if changes were made. The images or other third party material in this article are included in the article's Creative Commons licence, unless indicated otherwise in a credit line to the material. If material is not included in the article's Creative Commons licence and your intended use is not permitted by statutory regulation or exceeds the permitted use, you will need to obtain permission directly from the copyright holder. To view a copy of this licence, visit http://creativecommons.org/licenses/by/4.0/ The Creative Commons Public Domain Dedication waiver (http://creativecommons.org/publicdomain/zero/1.0/) applies to the data made available in this article, unless otherwise stated in a credit line to the data. 


\begin{abstract}
(Continued from previous page)
Results: With a median follow-up of 12.5 months, 145 of the 217 camrelizumab-treated patients (66.8\%) experienced RCCEP (all grade 1 or 2). RCCEP occurred on the skin surface, mainly on the skin surface of head, face, and trunk. RCCEP could be divided into 5 types including "red-nevus-like," "pearl-like," "mulberry-like," "patch-like," and "tumor-like," according to the morphological features. RCCEP biopsy and pathology showed capillary endothelial hyperplasia and capillary hyperplasia in dermis. Significant association between RCCEP occurrence with higher objective response rate was observed (19.3\% vs. 5.6\%; one-sided $p=0.0044)$. Compared with those without RCCEP, patients with RCCEP had prolonged progression-free survival (median PFS; 3.2 months vs. 1.9 months; onesided $p<0.0001$ ) and overall survival (median OS; 17.0 months vs. 5.8 months; one-sided $p<0.0001$ ). In multivariable analyses, the development of RCCEP was significantly associated with prolonged PFS and OS after adjusting for baseline covariates. In addition, the landmark analyses of PFS and OS were consistent with the unadjusted analysis.
\end{abstract}

Conclusions: RCCEP occurred on the skin surface and was an immune response of skin capillary endothelial cells. RCCEP occurrence positively associated with outcomes of camrelizumab in advanced HCC.

Keywords: Anti-PD-1 antibody, Camrelizumab, Reactive cutaneous capillary endothelial proliferation, Hepatocellular carcinoma, Immune-related adverse events

\section{Background}

Immune checkpoint inhibitors (ICIs), especially monoclonal antibodies targeting programmed cell death protein-1 (PD-1) or its ligand PD-L1, have achieved remarkable results in the treatment of a variety of malignancies [1-5]. However, ICIs may cause immune-related adverse events (irAEs) due to overactivation of the body's immune system, which has attracted great attention. IrAEs commonly occur in the skin, colon, endocrine organs, liver, and lung [6] and are mostly grade 1 or 2, but a few serious irAEs can lead to death [7]. Accordingly, several toxicity management guidelines for irAEs have been issued since 2017 [8-11].

Nivolumab and pembrolizumab achieved high and durable response with manageable safety profiles in patients with sorafenib experienced advanced hepatocellular carcinoma (HCC) in phase 2 clinical studies $[12,13]$. Another anti-PD-1 monoclonal antibody camrelizumab showed an objective response rate (ORR) of $14.7 \%$, a 6 month overall survival (OS) rate of $74.4 \%$, and a disease control rate (DCR) of $44.2 \%$ in a phase 2 study [14]. Based on the study, the China National Medical Products Administration (NMPA) approved camrelizumab as second-line standard of care for advanced HCC. Except for the common irAEs, a special irAE, namely, reactive cutaneous capillary endothelial proliferation (RCCEP), has been observed in patients treated with camrelizumab. This study reported the occurrence and clinicopathological characteristics of RCCEP, as well as its correlation with efficacy of camrelizumab.

\section{Methods}

\section{Study design and patients}

Data of this study was derived from a multicenter, randomized, open-label, two administration methods parallel- group, phase 2 clinical trial assessing camrelizumab in pre-treated advanced HCC patients that was conducted at 13 study sites in China. From November 15, 2016 to November 16, 2017, 217 patients received camrelizumab 3 $\mathrm{mg} / \mathrm{kg}$ either every 2 weeks (q2w) or every 3 weeks (q3w) and were included in this analysis. The main inclusion criteria included a histological or cytological diagnosis of advanced HCC; progressed on or were intolerant to prior systemic treatment, mainly with sorafenib and/or oxaliplatin-based chemotherapy; not amenable to surgery or local treatment; those with an Eastern Cooperative Oncology Group (ECOG) performance status of $0-1$; ChildPugh scores of 7 or less; at least one measurable lesion as defined by Response Evaluation Criteria in Solid Tumors (RECIST) version 1.1.

\section{Outcome and assessments}

Tumor response was assessed, both by investigators and blinded independent central review (BICR), as complete response $(\mathrm{CR})$, partial response $(\mathrm{PR})$, stable disease $(\mathrm{SD})$, and progressive disease (PD) based on the RECIST version 1.1. ORR that was defined as the proportion of patients with $\mathrm{CR}$ or PR, and DCR that was defined the proportion of patients with $\mathrm{CR}, \mathrm{PR}$, or $\mathrm{SD} \geq 6$ weeks. PFS was defined as the time from initial treatment administration to progression or death, whichever occurred first. OS was defined as the time from initial treatment administration to death. AEs were graded according to the National Cancer Institute Common Terminology Criteria for Adverse Events (NCI-CTCAE version 4.03), except RCCEP. The grading criteria for RCCEP was defined as follows: grade 1, nodule(s) with a maximum diameter of $\leq 10 \mathrm{~mm}$, with or without rupture and bleeding; grade 2, nodule(s) with a maximum diameter of $>10 \mathrm{~mm}$, with or without rupture and bleeding; grade 
3, generalized nodules throughout the body, which may be complicated by skin infection; grade 4 , multiple and generalized nodules, life-threatening condition; and grade 5, death. Occurrence, morphological characteristics, and regression time of RCCEP were closely observed and recorded. RCCEP remission was defined as regressions of all RCCEP lesions.

\section{Histopathological analysis}

The RCCEP lesion tissues were fixed with $10 \%$ neutral formaldehyde, dehydrated with an alcohol gradient, infiltrated with paraffin wax, and embedded in paraffin to form paraffin-embedded blocks. Sections $(4 \mu \mathrm{m})$ were cut for hematoxylin and eosin (H\&E) staining, immunohistochemical (IHC), and immunofluorescence staining (Multiplex IF). The sections were baked at $60^{\circ} \mathrm{C}$ overnight, and then IHC and Multiplex IF staining were carried out using a Bond RX automatic stainer (Leica Biosystems, Buffalo Grove, IL). The stained sections were scanned using NanoZoomer Digital Pathology System (Nanozoomer 2.0-HT slide scanner (Hamamatsu, Hamamatsu City, Japan). The NDP view imaging software (NDP scan software) was used to analyze the scan results and catch representative images.

\section{Statistical analyses}

Data were analyzed using SAS version 9.4. Median duration of RCCEP was estimated with Kaplan-Meier method, and its $95 \%$ confidence interval (CI) was calculated with Brookmeyer-Crowley method. Correlation between RCCEP occurrence and ORR per BICR was analyzed using Fisher's exact test. Log-rank test was used to compare the PFS or OS distribution between patients who experienced RCCEP and those who did not. Medians were estimated with Kaplan-Meier method, and their 95\% CIs were calculated with the BrookmeyerCrowley method. Cox proportional-hazards model was used to assess the associations of RCCEP occurrence with PFS per BICR and OS and to calculate the hazard ratios (HRs) of PFS and OS for patients with or without RCCEP. Additional Cox model analysis was performed with extrahepatic metastasis (yes vs. no), portal vein invasion (yes vs. no), ECOG Performance Status (0 vs. 1), AFP level $(\geq 400 \mathrm{ng} / \mathrm{ml} \mathrm{vs.}<400 \mathrm{ng} / \mathrm{ml})$, and lines of previous treatment $(\geq 3$ vs. $<3$ ) as covariates. The RCCEP occurrence showed time-dependence, and thus associations of RCCEP occurrence with PFS per BICR and OS were also evaluated using landmark analysis to minimize lead-time bias. For PFS, the cutoff time point considered for landmark analysis was 1.5 months after the first dose. For OS, the cutoff time points considered for landmark analysis were 3.0 months and 5.0 months after the first dose. Landmark survival analyses only included patients who had not experienced PFS or OS events as of the cutoff time points.
Log-rank test was used to compare the PFS or OS distribution between patients who experienced RCCEP and those who did not as of the cutoff time point, and Cox proportional-hazards model was also used.

\section{Results \\ RCCEP occurrence, therapeutical approaches, and outcome}

With a median duration of follow-up of 12.5 months (range, $0.7-23.5$ months), 145 of 217 patients (66.8\%) experienced RCCEP, the most common AE related to camrelizumab. All RCCEP were grade 1 (117 patients [53.9\%]) or 2 (28 [12.9\%]). Among the 217 patients, 106 (48.8\%) had the first RCCEP occurrence during the first treatment cycle, whereas only 39 (18.0\%) had the first occurrence of during the second cycle or later. The median time to onset of RCCEP was 4.1 weeks (range, 0.129.1). There were no reports of visceral bleeding or death caused by RCCEP. No patients interrupted or discontinued treatment due to RCCEP.

Only in rare cases, RCCEP could be complicated by reactive capillary endothelial proliferation in other sites, including oral cavity (three [1.4\%]; two in the oral mucosa and one in the gingivae), eyes (two [0.9\%]; conjunctiva), and nasal cavity (two [0.9\%]; nasal mucosa) (see Additional file 1). The majority of reactive capillary endothelial proliferation in sites other than skin were grade 1 or 2 , except one (0.5\%), grade 3 reactive capillary endothelial proliferation in the nasal mucosa (see Additional file 1). No patients discontinued camrelizumab due to reactive capillary endothelial proliferation in sites other than skin. Only one patient experienced treatment interruption due to capillary endothelial proliferation in oral mucosa (grade 2).

Totally, 31 of the 145 patients (21.4\%) received symptomatic therapy for RCCEP, including laser therapy $(18,12.4 \%)$, minor resection (14, 9.7\%), hemostatic therapy (nine, 6.2\%), local hormone therapy (six, 4.1\%), systemic antibiotic treatment (two, 1.4\%), and cryotherapy (one, less than 1\%).

The median duration of RCCEP was 5.6 months (range, 0.9-22.7). As of the data cutoff date, RCCEP in 86 of the 145 patients $(59.3 \%$ ) achieved remission, with a median time to remission of 4.0 months (range, 0.917.1). In 26 of the 86 patients (30.2\%), all RCCEP lesions regressed on camrelizumab treatment, while in 60 patients (69.8\%), at least one RCCEP lesion regressed or necrosed and fell off after discontinuation of camrelizumab treatment with a median duration from last dose to remission was 1.6 months (range, 0.3-5.3). Among the 145 patients who experienced RCCEP, 32 patients $(22.1 \%)$ had RCCEP at the date of last dose and did not had a record of RCCEP remission at date cutoff. 
Morphological classification and characteristics of RCCEP RCCEP occurred on the skin surface, mainly on the skin of the head, face, and trunk. It could be divided into 5 types according to the morphology, namely, "red-nevuslike," "pearl-like," "mulberry-like," "patch-like," and "tumor-like," with "red-nevus-like" and "pearl-like" being the most common types (Fig. 1). Various types of RCCEP could be observed in the same patient. Initially, the lesions were usually bright red spots with a diameter of $\leq 2 \mathrm{~mm}$ ("red-nevus-like"). A few lesions were "patchor mulberry-like," and some "red-nevus-like" lesions gradually developed into "pearl-like" nodules with bright or dark red coloration, which tended to rupture and bleed, while a few of these "pearl-like" nodules developed into "tumor-like" nodules (diameter $>10 \mathrm{~mm}$ ). Most patients experienced RCCEP 2 to 4 weeks after the first dose. As the dose of medication increased, nodules became larger, increased in number, and gradually expanded in scope. Most skin nodules ceased to increase 3 to 4 months after the first dose. Some nodules gradually shrank, dried, and turned black. Some formed pedicled nodules that fell off spontaneously without leaving obvious scars.

\section{Pathological features of RCCEP}

The histopathological features of "red-nevus-like" lesions were as follows: the lesions were located in the reticular layer of dermis; the hyperplastic capillaries were sparsely arranged; and the lining endothelial cells were enlarged without atypia and were all mono-layered (Fig. 2a). The pathological manifestations of "pearl- or tumor-like" lesions were as follows: the lesions were located in the reticular layer of dermis and consisted of hyperplastic capillaries; the capillaries were arranged in lobulated or nodular pattern; fibrous interlobular connective tissue and intralobular or interlobular nutrient vessels with a large lumen were found in some cases; and interstitial fibrosis occurred in some cases (Fig. 2b). IHC staining showed intense staining of endothelial cells (CD31) as well as proliferation and division of endothelial cells (Ki67). High expressions of vascular endothelial growth factor-A (VEGF-A) and VEGFR2-pY1175 in the lesion tissues were detected (Fig. 3a). IF co-staining on the RCCEP biopsy tissue showed that a large number of $\mathrm{CD}^{+} \mathrm{T}$ cells, not $\mathrm{CD}^{+} \mathrm{T}$ cells, appeared around the capillaries of the lesion tissue. There was a high expression of the Th2 cytokine IL-4. CD163 ${ }^{+}$M2 macrophages were detected in the lesion tissue, and co-location of VEGF-A and $\mathrm{CD}_{163}{ }^{+} \mathrm{M} 2$ macrophages was identified (Fig. 3b).

\section{Correlation between RCCEP and the efficacy of camrelizumab}

Per BICR, 28 of 145 patients with RCCEP achieved PR. However, among the 72 patients without RCCEP, only 4 achieved PR. No patient achieved CR. Significant association between RCCEP occurrence with higher ORR was observed (19.3\% [95\% CI, 13.2-26.7] vs. 5.6\% [95\% CI, 1.5-13.6]; one-sided $p=0.0044)$. The ORR was $28.6 \%$ (95\% CI, 13.2-48.7) in patients who had grade 2 RCCEP and $17.1 \%$ (95\% CI, 10.8-25.2) in patients who had grade 1 RCCEP.

Significant associations between RCCEP occurrence with prolonged PFS and OS were observed. The median PFS was 3.2 months (95\% CI, 2.1-4.4) in patients with RCCEP and 1.9 months [95\% CI, 1.8-2.0] in patients without RCCEP (HR, 0.53 (95\% CI, 0.38-0.72) for progression or death; one-sided log-rank test $p<0.0001$; Fig. 4a). The median OS was 17.0 months (95\% CI, 14.4-not reached) in patients with RCCEP and 5.8 months [95\% CI, 4.0-7.3] in patients without RCCEP (HR, 0.33 (95\% CI, 0.22-0.47) for death; one-sided log-rank test $p<0.0001$; Fig. 4b). The median OS in patients who had grade 1 RCCEP was 16.1 months (95\% CI, 12.9-not reached), while that in patients who had grade 2 RCCEP was not reached (95\% CI, 16.2 months-not reached).

Additional Cox model analysis showed that RCCEP occurrence was still significantly correlated with PFS and OS after adjustment for baseline variables (HR for progression or death, 0.53 [95\% CI, 0.39-0.73]; HR for death, 0.33 [95\% CI, 0.23-0.47).

The median duration of camrelizumab treatment in patients with vs. without RCCEP was 5.5 months (range, $0.7-23.5$ ) vs. 1.5 months (range, less than 0.1-16.2).

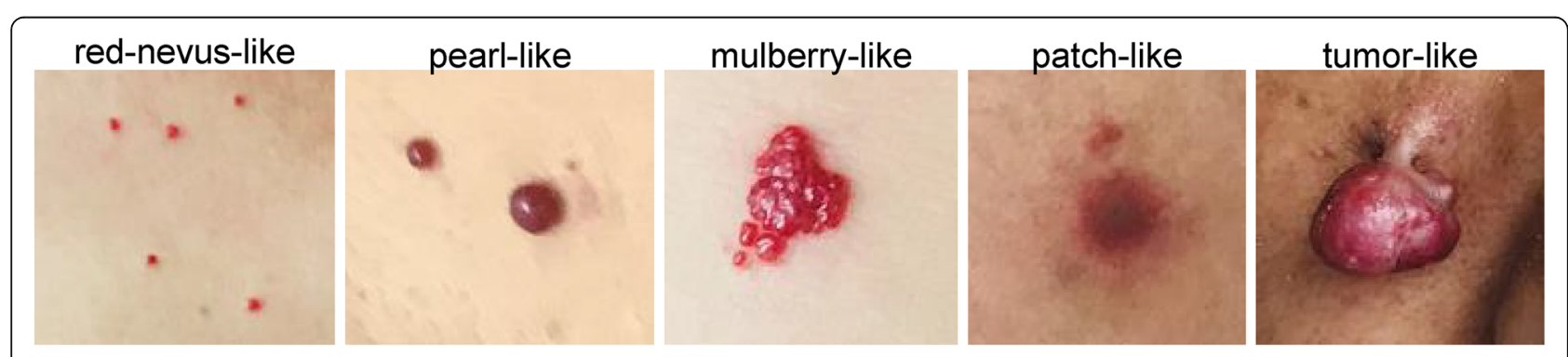

Fig. 1 Morphological classification of reactive cutaneous capillary endothelial proliferation 


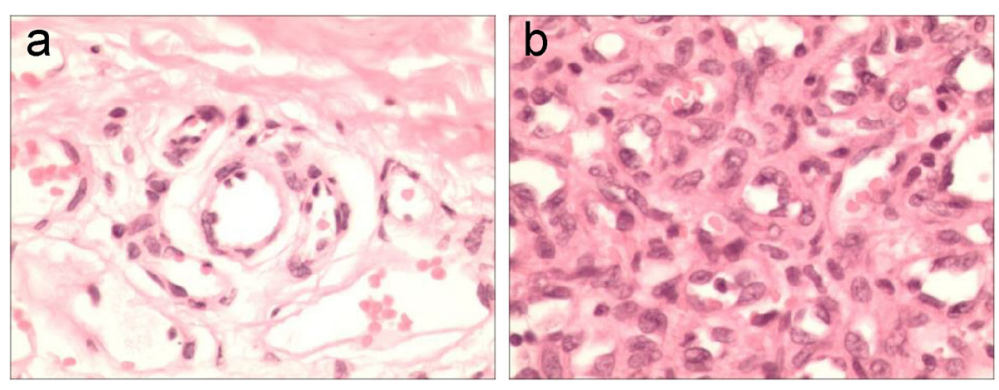

Fig. 2 Pathological characteristics of RCCEP. a "red-nevus-like" RCCEP pathological tissue. b "pearl-like" or "tumor-like" RCCEP pathological tissue. Tissues were analyzed using hematoxylin and eosin (H\&E) staining $(\times 400)$. RCCEP, reactive cutaneous capillary endothelial proliferation
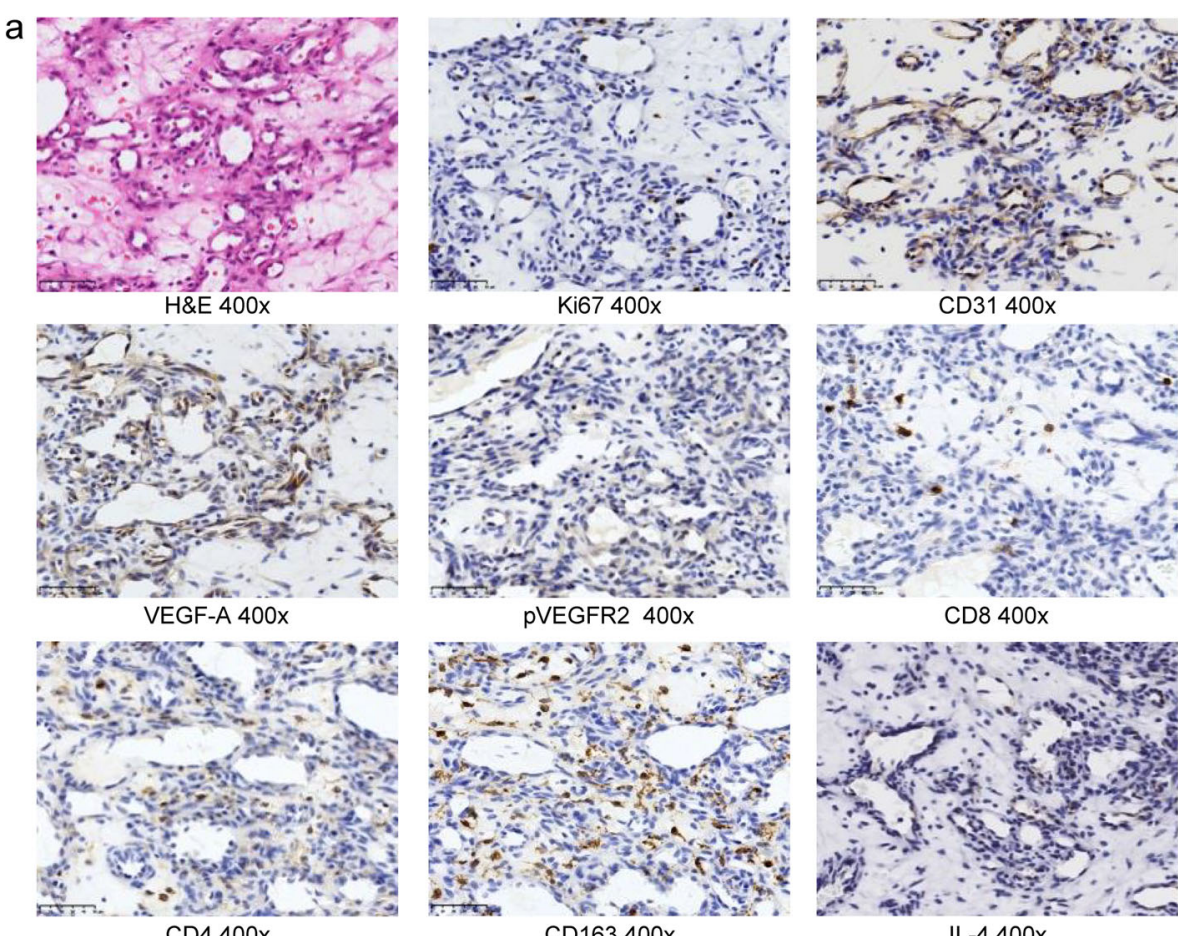
PVEGFR2 $400 x$
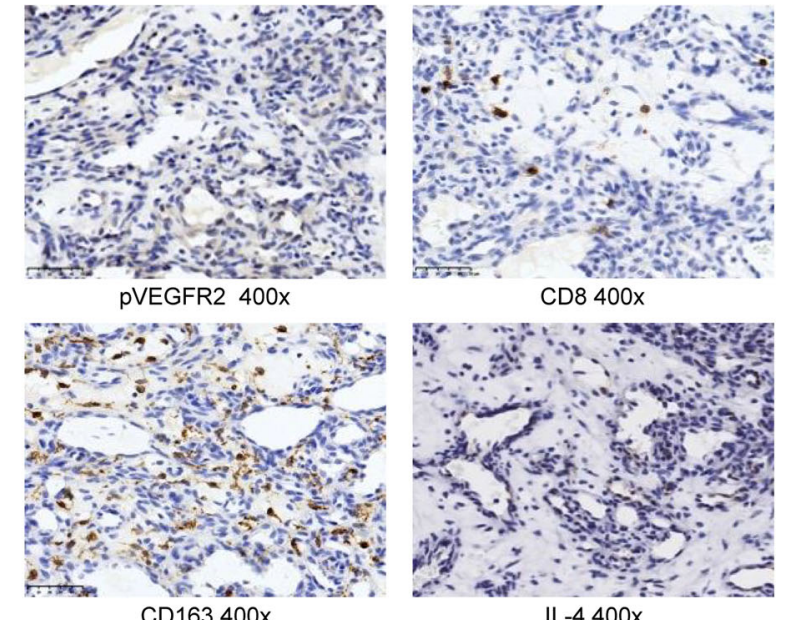

$$
\text { CD8 400x }
$$
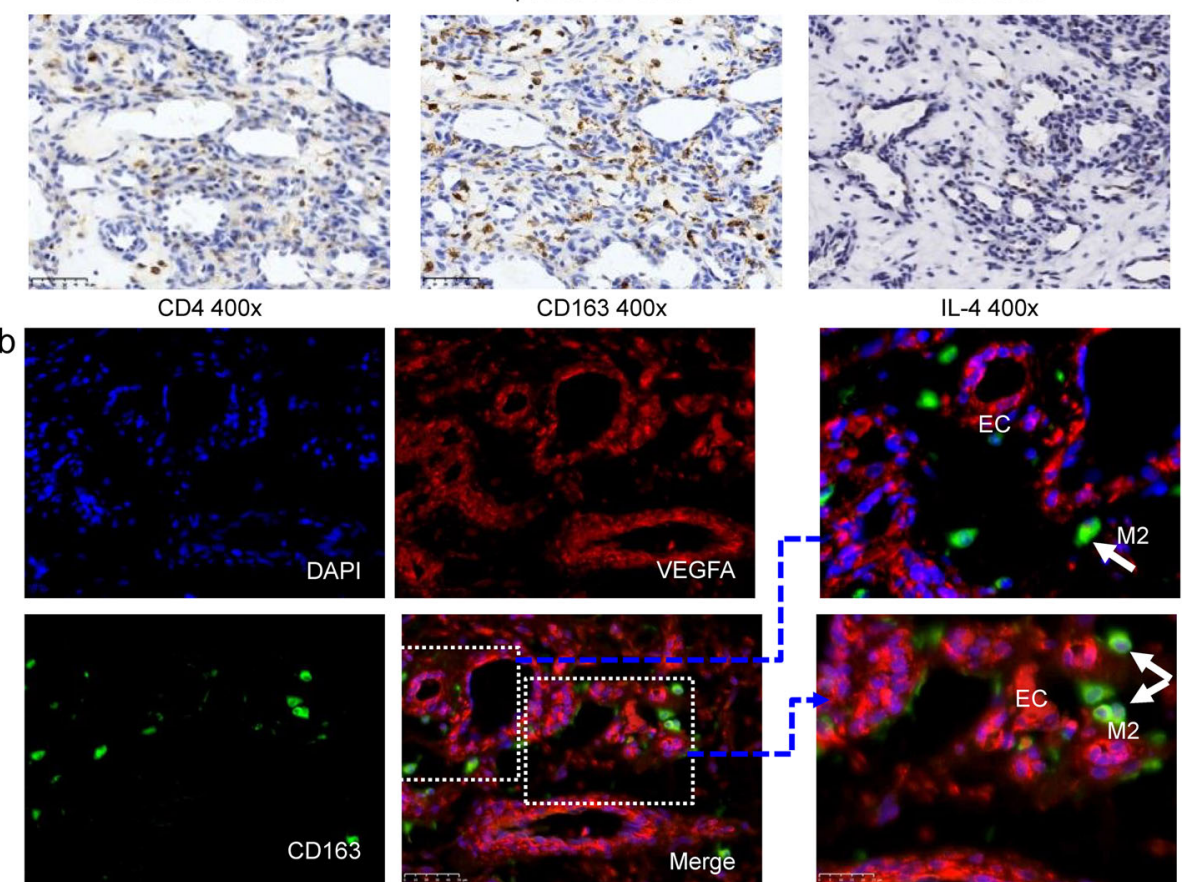

Fig. 3 "Pearl-like" RCCEP skin nodule histopathology and molecular marker detection. a Immunohistochemistry. b Immunofluorescence costaining (red, VEGF-A; green, CD163; and blue, DAPI. Arrow: M2 macrophages expressing VEGF-A). RCCEP, reactive cutaneous capillary endothelial proliferation 
a

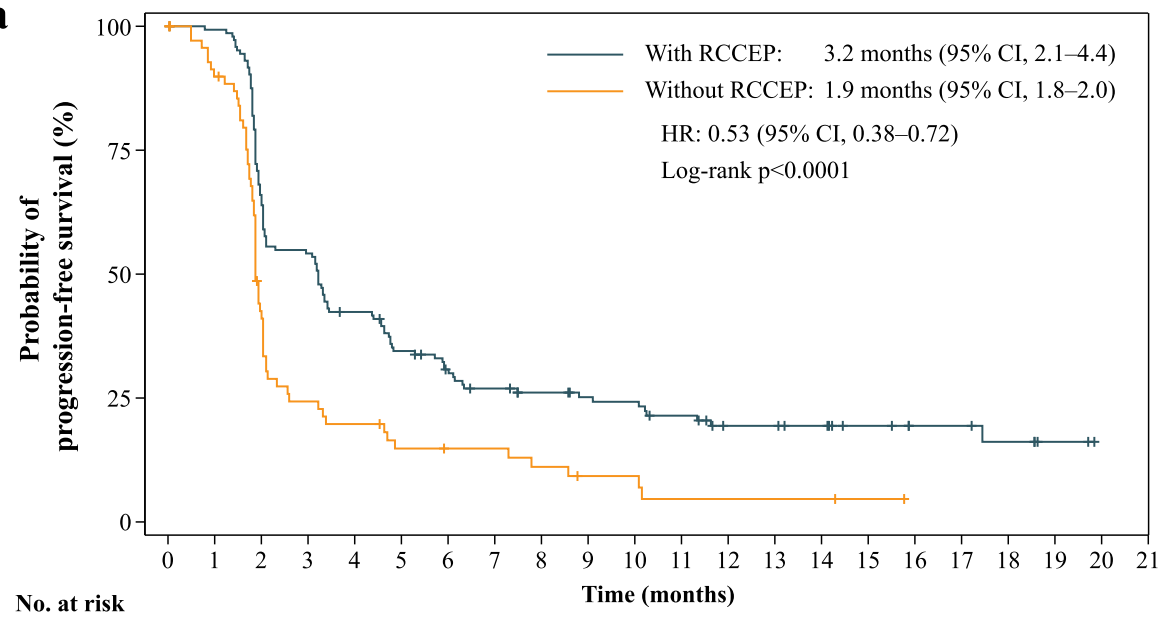

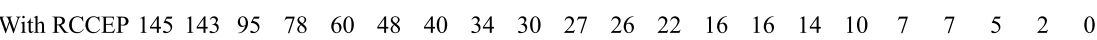
$\begin{array}{llllllllllllllllll}\text { Without RCCEP } & 72 & 62 & 28 & 16 & 13 & 9 & 8 & 8 & 6 & 4 & 4 & 2 & 2 & 2 & 2 & 1 & 0\end{array}$

b

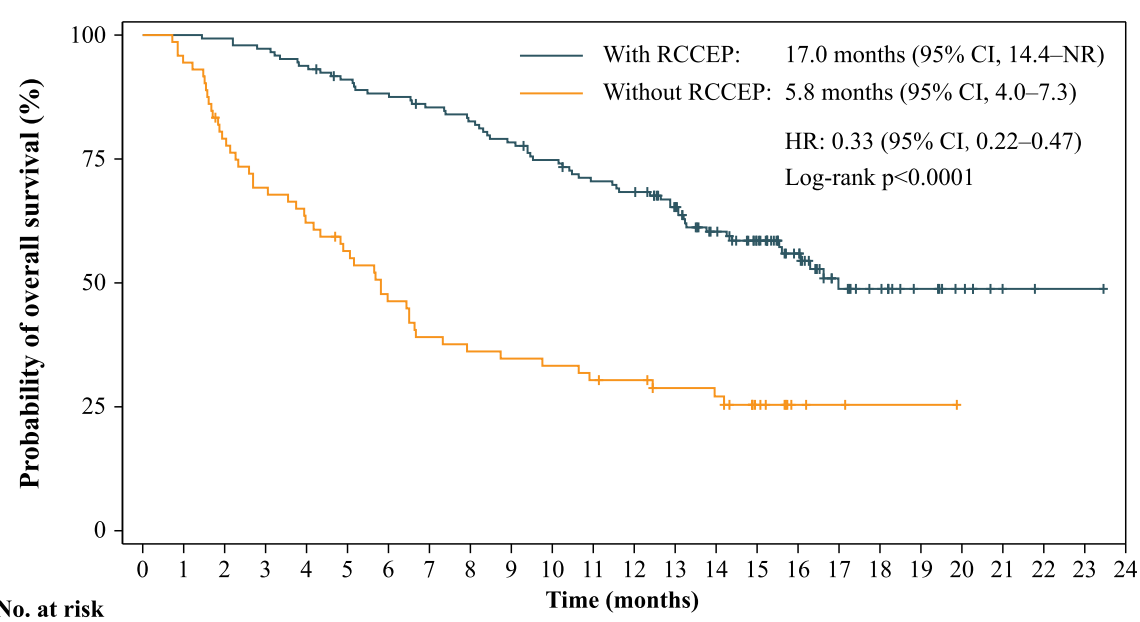

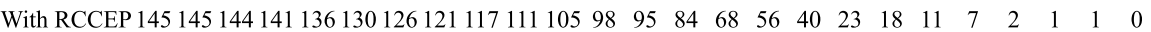

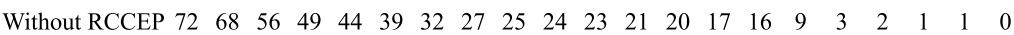

Fig. 4 Comparisons in survival between patients with and without RCCEP. a Kaplan-Meier curve of PFS. b Kaplan-Meier curve of OS. All 217 patients treated with camrelizumab were included in the analysis. RCCEP, reactive cutaneous capillary endothelial proliferation; PFS, progressionfree survival; OS, overall survival

Landmark survival analyses were conducted to take account of the time-dependence nature of RCCEP. The results further supported the significant associations between the RCCEP occurrence with PFS and OS. The landmark analysis of PFS at 1.5 months showed that the HR for progression or death of patients with RCCEP vs. patients without RCCEP at 1.5 months was 0.72 (95\% CI, 0.53-0.99; one-sided log-rank test $p=0.0363$; Fig. $5 a)$. Landmark survival analysis of OS at 3 and 5 months showed that the HRs for death of patients with RCCEP vs. patients without RCCEP at 3 and 5 months were 0.57 (95\% CI, 0.37-0.88; one-sided logrank test $p=0.0101$; Fig. $5 \mathrm{~b}$ ) and 0.58 (95\% CI, 0.350.97; one-sided log-rank test $p=0.0351$; Fig. 5c), respectively.

\section{Discussion}

In recent years, a number of clinical studies have been conducted on the treatment of advanced HCC with antiPD-1/PD-L1 monoclonal antibodies. Nivolumab and pembrolizumab have been conditionally approved by the U.S. Food and Drug Administration as second-line treatments for advanced HCC. However, the KEYNOTE-240 phase 3 study found no statistical difference in OS and PFS between pembrolizumab and placebo combined with the best support treatment [15]. The CheckMate459 study was a phase 3 study of nivolumab vs. sorafenib as a first-line treatment for advanced HCC, and there was no statistical difference between the two therapies in the primary endpoint (i.e., OS) [16]. It is encouraging that the results of the IMbrave 150 phase 3 study 


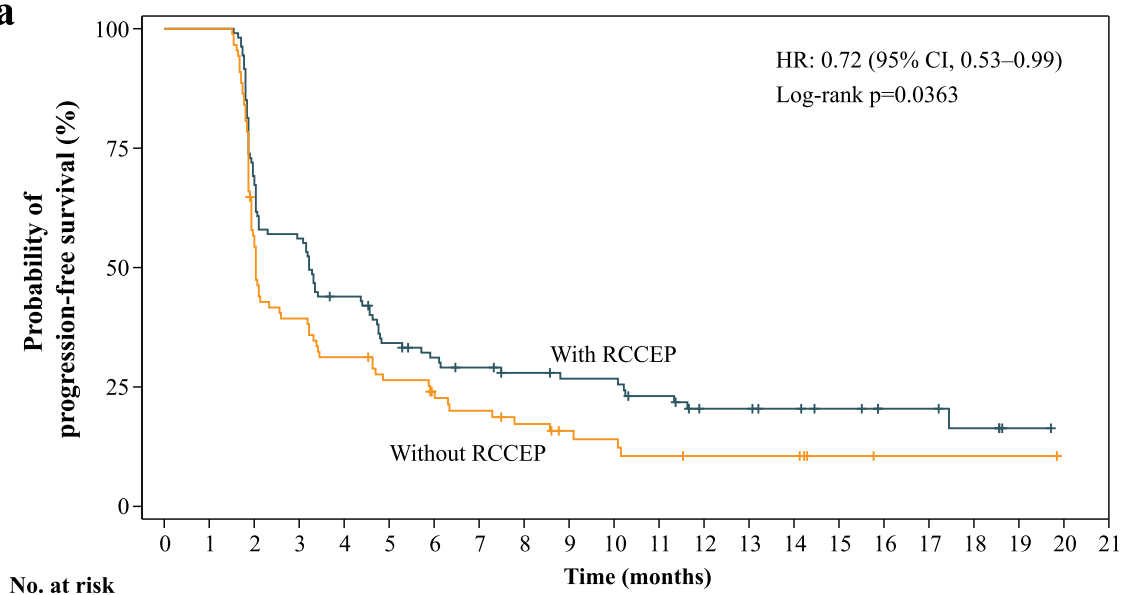

With RCCEP $107 \begin{array}{lllllllllllllllllllll}107 & 74 & 60 & 46 & 35 & 30 & 27 & 24 & 22 & 22 & 18 & 13 & 13 & 11 & 9 & 6 & 6 & 4 & 1 & 0\end{array}$ Without RCCEP $88 \begin{array}{lllllllllllllllllllll} & 88 & 49 & 34 & 27 & 22 & 18 & 15 & 12 & 9 & 8 & 6 & 5 & 5 & 5 & 2 & 1 & 1 & 1 & 1 & 0\end{array}$

b

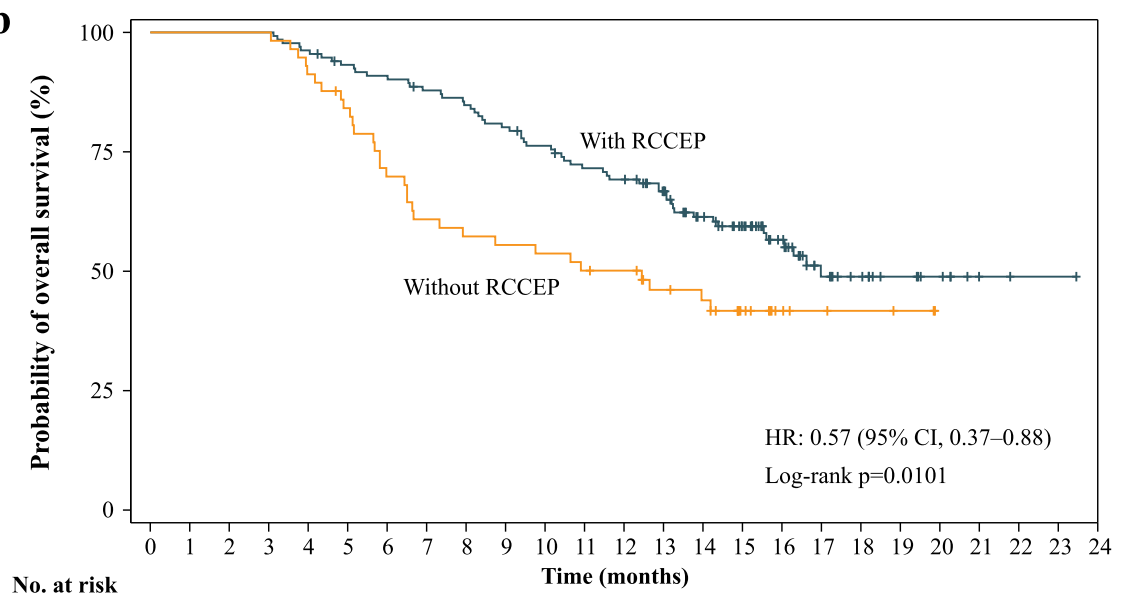

No. at risk Time (months)

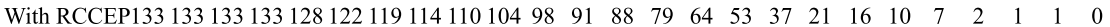

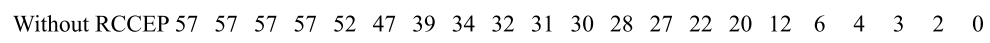

C

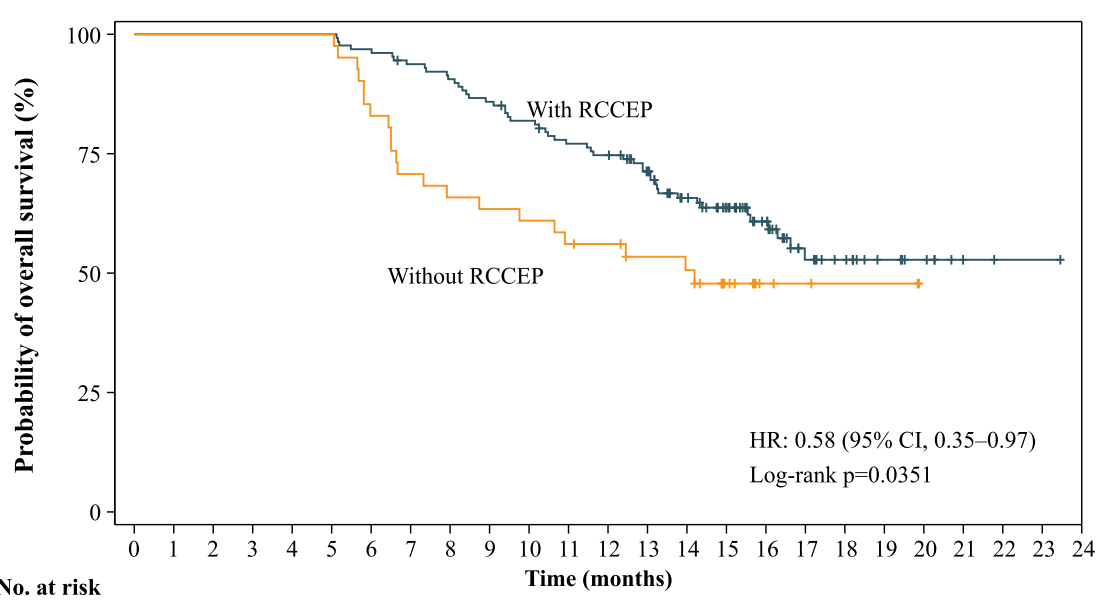

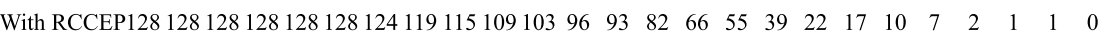

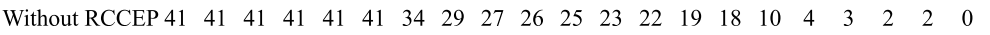

Fig. 5 (See legend on next page.) 
(See figure on previous page.)

Fig. 5 Landmark analysis of survival between patients with and without RCCEP. a Landmark survival analysis curve with 1.5 months as the cutoff time point. One hundred and ninety-five patients with PFS $\geq 1.5$ months were included in the analysis. The RCCEP group consisted of patients with RCCEP within 1.5 months and the group without RCCEP consisted of patients without RCCEP within 1.5 months. $\mathbf{b}$ Landmark survival analysis curve with 3.0 months as the cutoff time point. One hundred and ninety patients with OS $\geq 3.0$ months were included in the analysis. The RCCEP group consisted of patients with RCCEP within 3.0 months, and the group without RCCEP consisted of patients without RCCEP within 3.0 months. c Landmark survival analysis curve with 5.0 months as the cutoff time point. One hundred and sixty-nine patients with OS $\geq 5.0$ months were included in the analysis. The RCCEP group consisted of patients with RCCEP within 5.0 months, and the group without RCCEP consisted of patients without RCCEP within 5.0 months. RCCEP, reactive cutaneous capillary endothelial proliferation; PFS, progression-free survival; OS,

overall survival

comparing atezolizumab (an anti-PD-L1 antibody) plus bevacizumab vs. sorafenib as first-line treatment are positive (median OS, not reached with the combination therapy vs. 13.2 months with sorafenib, $p=0.0006$; median PFS, 6.8 vs. 4.3 months, $p<0.0001$ ) [17]. Combination of pembrolizumab and lenvatinib as well as nivolumab and ipilimumab in advanced HCC also has achieved encouraging results $[18,19]$.

ICIs can cause irAEs in multiple organs, with skin reactions being the most common. The skin irAEs approximately occur in $34 \%$ of patients treated with nivolumab or pembrolizumab [20] and usually do not require treatment discontinuation or dose reduction. Serious skin irAEs are rare. Besides common skin irAEs such as rash and pruritus, RCCEP is an irAE related to camrelizumab with the highest incidence. In the phase 1 clinical studies of camrelizumab monotherapy for the treatment of esophageal and nasopharyngeal carcinomas $[21,22]$, incidence of RCCEP were $76.7 \%$ (23/30) and $88 \%$ (82/93), respectively, but all events were grade 1 or 2 and did not lead to treatment discontinuation.

In this study, incidence of RCCEP was $66.8 \%$ (145/ 217 ), and the events mainly occurred on the skin of the head, face, and trunk. RCCEP is different from common skin rash, which has patchy distributions. Most of the RCCEP lesions were isolated and scattered on the skin. It is difficult to adopt the traditional grading criteria based on the covering area of body surface. Therefore, the grading standard for RCCEP was proposed based on the results of clinical studies and observation in clinical practices. This grading criterion has been adopted by the China NMPA and included in the label for camrelizumab. In this study, all RCCEP were grades 1 and 2, and no grades 3-5 RCCEP were observed.

Cutaneous hemangioma is a benign vasculopathy with various classifications. It usually occurs in infants, at birth or within 1 month after. It mostly invades the skin of the head and neck, but can also occur in the mucosa, liver, lower limbs, and muscles. It is sensitive to glucocorticoid treatment, and a few cases will spontaneously resolve after several months or years. RCCEP is different from common hemangioma in terms of pathogenesis, morphological manifestations, pathological features, treatment outcomes, and ways of remission. Therefore, RCCEP cannot be defined by the term "hemangioma." Pathological analysis of early-stage RCCEP showed an increased size of capillary endothelial cells and capillary hyperplasia in dermis; when it developed to "pearl-like" or "tumor-like" RCCEP, the capillaries obviously increased and showed a lobulated or nodular arrangement. Based on the IHC analysis, strong staining of CD31 indicated a large number of endothelial cells in the lesion tissue, and high expression of $\mathrm{Ki}-67$ indicated rapid proliferation of endothelial cells. High expressions of VEGF-A and VEGFR2-pY1175 demonstrated activation of angiogenesis via VEGFR2 signal pathway. Combined with the results of IF, camrelizumab might activate $\mathrm{CD}^{+}{ }^{+} \mathrm{T}$ cells, thus increasing Th2 cytokine IL-4, stimulating the differentiation of $\mathrm{CD}_{163^{+}} \mathrm{M} 2$ macrophages, and then promoting vascular proliferation by releasing VEGF-A.

Anti-VEGFR2 antibodies such as ramucirumab, tanibirumab, and CDP791 could cause grades 1 and 2 cutaneous capillary hyperplasia in clinical studies and lesions regressed after treatment were stopped. The VEGF-A concentration increased, and VEGFR-2 was widely expressed in the specimens of lesion tissues [23-25]. Hwang et al. reported that $49 \%(40 / 82)$ of patients with metastatic melanoma treated with nivolumab or pembrolizumab experienced skin AEs, among whom 2 cases experienced cutaneous capillary hyperplasia (2.4\%) [26]. Therefore, RCCEP may not be unique to camrelizumab, but with a high incidence and different manifestations. It is speculated that the reactivation of immune responses may destroy the dynamic balance between pro- and anti-angiogenic factors, and thus cause proliferation of skin capillary endothelial cells.

In a phase 1 study in advanced HCC, gastric cancer, and gastroesophageal junction cancer, the incidence of RCCEP in patients treated with camrelizumab combined with apatinib was only $12.1 \%$ (4/33), and all events were grades 1 and 2 [27]. Apatinib is a highly selective VEGFR2 inhibitor. It is presumed that it inhibited the development of RCCEP by blocking signal transduction after binding to VEGF. In a phase 1 study in nasopharyngeal carcinoma, the incidence of RCCEP with camrelizumab monotherapy was $88 \%$ (82/93), whereas the incidence with camrelizumab plus gemcitabine/cisplatin decreased to $22 \%(5 / 23)$ [22]. Chemotherapy may inhibit the RCCEP occurrence by affecting angiogenesis. 
Shi et al. reported that among the 20 ICI-treated patients who developed skin irAEs, four patients are complicated by oral mucosa lesions involving the tongue, buccal mucosa, lips, and/or gingivae, one showed papules, and three had erosions resembling oral lichen planus [28]. In this study, very few patients with RCCEP had lesions in the oral mucosa, gingivae, nasal mucosa, and palpebral conjunctiva. However, no occurrence in the tracheal or digestive (esophageal and gastrointestinal) mucosa was observed. Therefore, there is no risk of hemoptysis or gastrointestinal hemorrhage.

Skin irAEs could predict the efficacy of anti-PD-1 monoclonal antibodies in some solid tumors [29-31]. In this study, RCCEP occurrence was significantly correlated with response to camrelizumab. ORR was $19.3 \%$ vs. $5.6 \%$ in patients with vs. without RCCEP. In addition, significant associations were observed between RCCEP occurrence with prolonged PFS and OS, suggesting that patients with RCCEP had better prognoses. Therefore, RCCEP may become a clinical biomarker for predicting efficacy of camrelizumab.

Most RCCEP did not require special treatment. RCCEP was insensitive to glucocorticoids, and most lesions spontaneously resolved within 1.6 months after discontinuation of camrelizumab. If the nodule is large, and rupture and bleeding occur, Yunnan Baiyao powder can be given for local hemostasis, and mupirocin ointment can be given, if necessary, to prevent infection. Minor resection or laser treatment can be adopted for a few patients. If skin infection occurs, local or systemic antibiotic treatment should be conducted. When grade of RCCEP decreases to grade 1, camrelizumab administration can be resumed as appropriate.

The major limitation of this study is that mechanism of RCCEP occurrence is still unclear, but may be attributed to immune stress responses of the cutaneous capillary endothelial cells.

\section{Conclusion}

RCCEP was a common irAE of camrelizumab, which mainly occurred on the skin of the head, face, and trunk. It had unique morphological features and often spontaneously regressed or necrosed and fell off after treatment discontinuation. RCCEP occurrence was closely related to the objective response and PFS and OS benefits of camrelizumab treatment, which requires further investigations.

\section{Supplementary information}

Supplementary information accompanies this paper at https://doi.org/10. 1186/s13045-020-00886-2.

Additional file 1. Occurrence site and grade of reactive capillary endothelial proliferation.

\section{Abbreviations}

RCCEP: Reactive cutaneous capillary endothelial proliferation; ICl: Immune checkpoint inhibitor; PD-1: Programmed cell death protein-1;
HCC: Hepatocellular carcinoma; FDA: Food and Drug Administration; NMPA: National Medical Products Administration; ECOG: Eastern Cooperative Oncology Group; AE: Adverse event; irAE: Immune-related adverse event; BICR: Blinded independent central review; CR: Complete response; PR: Partial response; SD: Stable disease; PD: Progressive disease; ORR: Objective response rate; DCR: Disease control response; PFS: Progression-free survival; OS: Overall survival; $\mathrm{Cl}$ : Confidence interval; $\mathrm{IHC}$ : Immunohistochemistry; IF: Immunofluorescence; AFP: Alpha-fetoprotein

\section{Acknowledgements}

We thank the patients and their families and all members of the collaborative group on camrelizumab for treatment of HCC. We would also like to acknowledge Hui Dong, PhD (Medical Writer at Hengrui) for the medical writing support.

\section{Authors' contributions}

Concept and design: SQ, XS, FW, LW, PY, and JZ. Acquisition, analysis, or interpretation of data: all authors. Statistical analysis: FW and PY. Drafting of the manuscript: FW, SQ, XS, LW, and PY. Critical revision of the manuscript for important intellectual content: all authors. Approval of the final manuscript: all authors.

\section{Funding}

This study was supported by Jiangsu Hengrui Medicine Co., Ltd. The sponsor participated in the study design, data analysis and interpretation, and preparation and review of the manuscript in collaboration with the authors and funded data analysis. The sponsor participated in the decision to submit the manuscript for publication but had no right to veto that decision.

\section{Availability of data and materials}

All data generated or analyzed during this study are included in this published article and its supplementary information files.

\section{Ethics approval and consent to participate}

The protocol of the phase 2 study was reviewed and approved by the Ethics Committee of each study site. The study was conducted in compliance with the ethical principles of Good Clinical Practice and the requirements of local laws and regulations, as well as the Declaration of Helsinki and its amendments. Written informed consent was obtained from each patient.

\section{Consent for publication}

Not applicable

\section{Competing interests}

Linna Wang, Ping Yan, and Jianjun Zou are employees of Jiangsu Hengrui Medicine Co., Ltd. Other co-authors declare no competing interests.

\section{Author details}

${ }^{1}$ Department of Radiotherapy, The First Affiliated Hospital of Nanjing Medical College, No. 300, Guangzhou Rode, Nanjing 210029, China. ${ }^{2}$ Department of Medical Oncology, Cancer Center of Jinling Hospital, No. 34, 34 Biao, Yanggongjing Street, Nanjing 210002, China. ${ }^{3}$ Liver Cancer Institute, Zhongshan Hospital, Fudan University, Shanghai, China. ${ }^{4}$ Minimally Invasive Therapy Center, Fudan University Shanghai Cancer Center, Shanghai, China. ${ }^{5}$ Department of Medical Oncology, The Second Affiliated Hospital of Anhui Medical University, Hefei, China. ${ }^{6}$ Department of Intervention, Hunan Cancer Hospital, Changsha, China. ${ }^{7}$ Department of Medical Oncology, The First Affiliated Hospital of Nanchang University, Nanchang, China. ${ }^{8}$ Department of Medical Oncology, 3rd Affiliated Hospital of Harbin Medical University, Harbin, China. 'Department of Medical Oncology, National Cancer Center/ Cancer Hospital, Chinese Academy of Medical Sciences and Peking Union Medical College, Beijing, China. ${ }^{10}$ Department of Medical Oncology, West China Hospital, Sichuan University, Chengdu, Sichuan, China. ${ }^{11}$ Department of Medical Oncology, The First Affiliated Hospital of Zhejiang University School of Medicine, Hangzhou, China. ${ }^{12}$ Department of Medical Oncology, Fujian Medical University Union Hospital, Fuzhou, China. ${ }^{13}$ Department of Interventional Radiology, Cancer Center, Guangdong Provincial People's Hospital, Guangzhou, China. ${ }^{14}$ Department of Medical Oncology, First Affiliated Hospital of Xi'an Jiaotong University (School of Medicine), Xi'an, China. ${ }^{15}$ Clinical Research \& Development, Jiangsu Hengrui Medicine Co., Ltd, Shanghai, China. 
Received: 24 February 2020 Accepted: 28 April 2020

Published online: 11 May 2020

\section{References}

1. Rosenberg JE, Hoffman-Censits J, Powles T, van der Heijden MS, Balar AV, Necchi A, et al. Atezolizumab in patients with locally advanced and metastatic urothelial carcinoma who have progressed following treatment with platinum-based chemotherapy: a single-arm, multicentre, phase 2 trial. Lancet. 2016:387:1909-20.

2. Kaufman HL, Russell J, Hamid O, Bhatia S, Terheyden P, D'Angelo SP, et al. Avelumab in patients with chemotherapy-refractory metastatic Merkel cell carcinoma: a multicentre, single-group, open-label, phase 2 trial. Lancet Oncol. 2016:17:1374-85.

3. Robert C, Long GV, Brady B, Dutriaux C, Maio M, Mortier L, et al. Nivolumab in previously untreated melanoma without BRAF mutation. N Engl J Med. 2015;372:320-30

4. Herbst RS, Baas P, Kim DW, Felip E, Perez-Gracia JL, Han JY, et al. Pembrolizumab versus docetaxel for previously treated, PD-L1-positive, advanced non-small-cell lung cancer (KEYNOTE-010): a randomised controlled trial. Lancet. 2016:387:1540-50.

5. Planchard D, Yokoi T, McCleod MJ, Fischer JR, Kim YC, Ballas M, et al. A Phase III Study of Durvalumab (MED14736) With or without tremelimumab for previously treated patients with advanced NSCLC: rationale and protocol design of the ARCTIC study. Clin Lung Cancer. 2016;17:232-6.

6. Friedman CF, Proverbs-Singh TA, Postow MA. Treatment of the immunerelated adverse effects of immune checkpoint inhibitors: a review. JAMA Oncol. 2016;2:1346-53.

7. Wang DY, Salem JE, Cohen JV, Chandra S, Menzer C, Ye F, et al. Fatal Toxic effects associated with immune checkpoint inhibitors: a systematic review and meta-analysis. JAMA Oncol. 2018;4:1721-8.

8. Puzanov I, Diab A, Abdallah K, Bingham CO 3rd, Brogdon C, Dadu R, et al. Managing toxicities associated with immune checkpoint inhibitors: consensus recommendations from the Society for Immunotherapy of Cancer (SITC) Toxicity Management Working Group. J Immunother Cancer. 2017:5:95.

9. Haanen J, Carbonnel F, Robert C, Kerr KM, Peters S, Larkin J, et al. Management of toxicities from immunotherapy: ESMO Clinical Practice Guidelines for diagnosis, treatment and follow-up. Ann Oncol. 2018;29: iv264-iv6.

10. Brahmer JR, Lacchetti C, Schneider BJ, Atkins MB, Brassil KJ, Caterino JM, et al. Management of immune-related adverse events in patients treated with immune checkpoint inhibitor therapy: American Society of Clinical Oncology Clinical Practice Guideline. J Clin Oncol. 2018;36:1714-68.

11. Chinese Society of Clinical Oncology (CSCO). Management of immune checkpoint inhibitor-related toxicity, vol. V1. Beijing: People's Health Publishing House; 2019.

12. Zhu AX, Finn RS, Edeline J, Cattan S, Ogasawara S, Palmer D, et al. Pembrolizumab in patients with advanced hepatocellular carcinoma previously treated with sorafenib (KEYNOTE-224): a non-randomised, openlabel phase 2 trial. Lancet Oncol. 2018;19:940-52.

13. El-Khoueiry AB, Sangro B, Yau T, Crocenzi TS, Kudo M, Hsu C, et al. Nivolumab in patients with advanced hepatocellular carcinoma (CheckMate 040): an open-label, non-comparative, phase 1/2 dose escalation and expansion trial. Lancet. 2017:389:2492-502.

14. Qin S, Ren Z, Meng Z, Chen Z, Chai X, Xiong J, et al. Camrelizumab in patients with previously treated advanced hepatocellular carcinoma: a multicentre, open-label, parallel-group, randomised, phase 2 trial. Lancet Oncol. 2020;21:571-80.

15. Finn RS, Ryoo B-Y, Merle $P$, Kudo $M$, Bouattour $M$, Lim H-Y, et al. Results of KEYNOTE-240: phase 3 study of pembrolizumab (Pembro) vs best supportive care (BSC) for second line therapy in advanced hepatocellular carcinoma (HCC). 2019; 37:4004.

16. Yau T, Park JW, Finn RS, Cheng A-L, Mathurin P, Edeline J, et al. LBA38 CheckMate 459: a randomized, multi-center phase III study of nivolumab (NIVO) vs sorafenib (SOR) as first-line (1 L) treatment in patients (pts) with advanced hepatocellular carcinoma (aHCC). Ann Oncol. 2019;30:mdz394.029.

17. Cheng AL, Qin S, Ikeda M, Galle P, Ducreux M, Zhu A, et al. LBA3 IMbrave150: Efficacy and safety results from a ph III study evaluating atezolizumab (atezo) + bevacizumab (bev) vs sorafenib (Sor) as first treatment (tx) for patients (pts) with unresectable hepatocellular carcinoma (HCC). Ann Oncol. 2019;30:mdz446.002.
18. Ikeda M, Sung MW, Kudo M, Kobayashi M, Baron AD, Finn RS, et al. 747P a phase Ib trial of lenvatinib (LEN) plus pembrolizumab (PEMBRO) in unresectable hepatocellular carcinoma (uHCC): updated results. Ann Oncol. 2019;30:mdz247.073.

19. Yau T, Kang Y-K, Kim T-Y, El-Khoueiry AB, Santoro A, Sangro B, et al. Nivolumab (NIVO) + ipilimumab (IPI) combination therapy in patients (pts) with advanced hepatocellular carcinoma (aHCC): results from CheckMate 040. 2019; 37:4012.

20. Belum VR, Benhuri B, Postow MA, Hellmann MD, Lesokhin AM, Segal NH, et al. Characterisation and management of dermatologic adverse events to agents targeting the PD-1 receptor. Eur J Cancer. 2016;60:12-25.

21. Huang J, Xu B, Mo H, Zhang W, Chen X, Wu D, et al. Safety, activity, and biomarkers of SHR-1210, an anti-PD-1 antibody, for patients with advanced esophageal carcinoma. Clin Cancer Res. 2018;24:1296-304.

22. Fang W, Yang Y, Ma Y, Hong S, Lin L, He X, et al. Camrelizumab (SHR-1210) alone or in combination with gemcitabine plus cisplatin for nasopharyngeal carcinoma: results from two single-arm, phase 1 trials. Lancet Oncol. 2018; 19:1338-50.

23. Spratlin JL, Mulder KE, Mackey JR. Ramucirumab (IMC-1121B): a novel attack on angiogenesis. Future Oncol. 2010;6:1085-94.

24. Lee SJ, Lee SY, Lee WS, Yoo JS, Sun JM, Lee J, et al. Phase I trial and pharmacokinetic study of tanibirumab, a fully human monoclonal antibody to vascular endothelial growth factor receptor 2, in patients with refractory solid tumors. Invest New Drugs. 2017;35:782-90.

25. Ton NC, Parker GJ, Jackson A, Mullamitha S, Buonaccorsi GA, Roberts C, et al. Phase I evaluation of CDP791, a PEGylated di-Fab' conjugate that binds vascular endothelial growth factor receptor 2. Clin Cancer Res. 2007; 13:7113-8.

26. Hwang SJ, Carlos G, Wakade D, Byth K, Kong BY, Chou S, et al. Cutaneous adverse events (AEs) of anti-programmed cell death (PD)-1 therapy in patients with metastatic melanoma: a single-institution cohort. J Am Acad Dermatol. 2016;74:455-61.

27. Xu J, Zhang Y, Jia R, Yue C, Chang L, Liu R, et al. Anti-PD-1 antibody SHR1210 combined with apatinib for advanced hepatocellular carcinoma, gastric, or esophagogastric Junction Cancer: An Open-label, Dose Escalation and Expansion Study. Clin Cancer Res. 2019;25:515-23.

28. Shi VJ, Rodic N, Gettinger S, Leventhal JS, Neckman JP, Girardi M, et al, Clinical and histologic features of lichenoid mucocutaneous eruptions due to anti-programmed cell death 1 and anti-programmed cell death ligand 1 immunotherapy. JAMA Dermatol. 2016;152:1128-36.

29. Hua C, Boussemart L, Mateus C, Routier E, Boutros C, Cazenave H, et al. Association of vitiligo with tumor response in patients with metastatic melanoma treated with pembrolizumab. JAMA Dermatol. 2016;152:45-51.

30. Freeman-Keller M, Kim Y, Cronin H, Richards A, Gibney G, Weber JS. Nivolumab in resected and unresectable metastatic melanoma: characteristics of immune-related adverse events and association with outcomes. Clin Cancer Res. 2016;22:886-94.

31. Sanlorenzo M, Vujic I, Daud A, Algazi A, Gubens M, Luna SA, et al. Pembrolizumab cutaneous adverse events and their association with disease progression. JAMA Dermatol. 2015;151:1206-12.

\section{Publisher's Note}

Springer Nature remains neutral with regard to jurisdictional claims in published maps and institutional affiliations.

Ready to submit your research? Choose BMC and benefit from:

- fast, convenient online submission

- thorough peer review by experienced researchers in your field

- rapid publication on acceptance

- support for research data, including large and complex data types

- gold Open Access which fosters wider collaboration and increased citations

- maximum visibility for your research: over $100 \mathrm{M}$ website views per year

At $\mathrm{BMC}$, research is always in progress.

Learn more biomedcentral.com/submissions 